\title{
Feedforward Control Analytical Model in SPC
}

\author{
Cui Youxiang ${ }^{1}$, Hong Wei ${ }^{2}$, Li Jianshe ${ }^{3}$, Ju Xiaoming ${ }^{4}$ \\ ${ }^{1}$ An Tai College of Economics \& management, Shanghai Jiao Tong University, Shanghai 200052, China \\ ${ }^{2}$ Human resources department, power supply company of Tiantai county Zhejiang province 318000 , China \\ ${ }^{3}$ Shanghai Broad Vision information Science and Technology Co. Ltd. Shanghai 200092, China \\ ${ }^{4}$ Software Engineering Institute, East China Normal University, Shanghai 200062, China \\ cuiyouxiang@aliyun.com
}

\begin{abstract}
When there are inherent disturbances or noise in the system inputs that are impossible or impractical to remove, various forms of feedback and feedforward adjustment have been used in the Statistic Process Control (SPC). This article presents a general feedforward control framework to analysis and compensate for deviations in the quality characteristic.

Index Terms - Feedforward control, SPC Quality managemen
\end{abstract}

\section{Introduction}

To the statistician, the words "process control" have usually meant the quality control techniques developed originally by Shewhartin the United States. Many of the techniques developed by mathematical statisticians, physicists, chemists, engineers, and others for the analysis of data may be used in the control of product quality. The expression statistical process control may be used to cover all uses of statistical techniques for this purpose.

More recently, the sequential aspects of quality control have been emphasized, leading to the introduction of cumulative sum charts by Page and Barnard and the geometric moving average charts of Roberts. Such charts are frequently employed in industries concerned with the manufacture of discrete "parts" as one aspect of what is called statistical process control (SPC). In particular, they are used for continuous monitoring of a process. That is, they are used to supply a continuous screening mechanism for detecting assignable (or special) causes of variation. Appropriate display of plant data ensures that significant changes are quickly brought to the attention of those responsible for running the process. Knowing the answer to the question "when did a change of this particular kind occur?" we may be able to answer the question "why did it occur?" Hence a continuous incentive for process stabilization and improvement can be achieved.

When we can measure fluctuations in an input variable that can be observed but not changed, it may be possible to make appropriate compensatory changes in some other control variable. This is referred to as feedforward control.

\section{Modeling the Feedback Loop}

General system of feedback control is shown in Figure1.

The process is affected by a disturbance that in the absence of compensatory action would cause the output quality characteristic to deviate from target by an amount $N_{t}$. Thus, $\left\{N_{t}\right\}$ is a time series exemplifying what would happen at the output if no control were applied. In fact, a compensating variable $X_{t}$ (deposition rate in our example) can be manipulated to cancel out this disturbance as far as possible. Changes in $X$ will pass through the process and be acted on by its dynamics to produce at time $t$ an amount of compensation $Y_{t}$ at the output (again measured as a deviation from target). To the extent that this compensation $Y_{t}$ fails to cancel out the disturbance $N_{t}$, there will be an error, or deviation from target $\varepsilon_{\mathrm{t}}=\mathrm{Y}_{\mathrm{t}}-\mathrm{T}$, equal to $\varepsilon_{\mathrm{t}}=\mathrm{N}_{\mathrm{t}}+\mathrm{Y}_{\mathrm{t}}$. The controller is some means (automatic or manual) that brings into effect the control equation $X_{t}=f\left(\varepsilon_{t}, \varepsilon_{t-1}, \ldots\right)$, which adjusts the output depending on present and past errors.

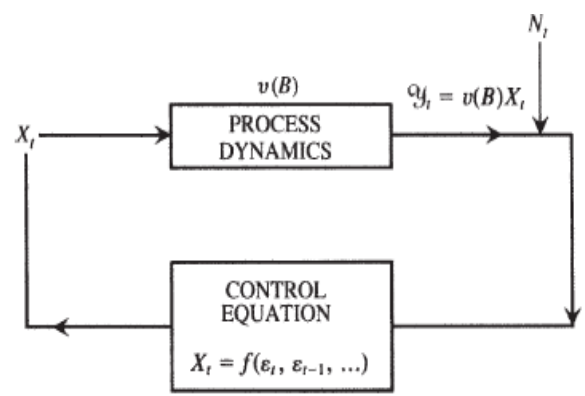

Fig. 1 Feedback control loop

\section{Design of Discrete Feedforward Control Schemes}

A common adjustment problem is to maintain an output variable close to a target value in a dynamic system subject to disturbances by manipulation of an input variable, to obtain feedback control. Feedback control schemes use only the observed deviation of the output from target as a basis for adjustment of the input variable

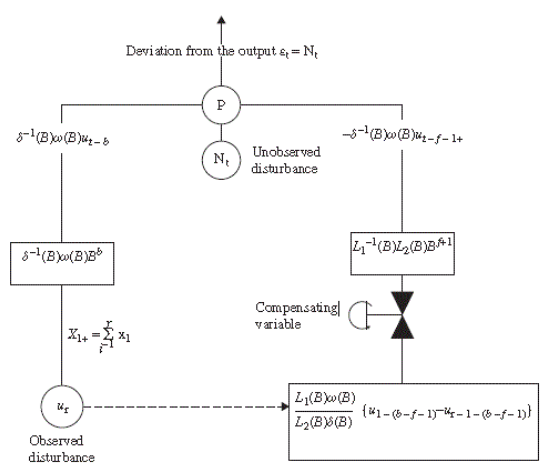

Fig. 2: System at time $t$ subject to an observed input disturbance $u t$ and unobserved disturbance $N t$, with potential compensating variable $X t$ [1] 
We now consider the design of discrete feedforward control schemes that give minimum mean square error at the output. A situation arising in the manufacture of a polymer is illustrated in Figure 2. The $\mathrm{v} Y_{t}$ of the product is known to vary in part due to fluctuations $u_{t}$, which can be observed but not changed. The $X_{t}$ is a control variable that is measured, can be manipulated, and is potentially available to alter the viscosity by any desired amount and hence compensate potential deviations from target. The total effect in the output viscosity of all other sources of disturbance at time $t$ is denoted by $N$.

\subsection{Minimize Mean Square Error at the Output}

Suppose that $Y_{t}, u_{t}, X_{t}, N_{t}$ are deviations from reference values, which are such that if the conditions $u=0, X=0, N=0$ were continuously maintained, the process would remain in an equilibrium state such that the output was exactly on the target value $Y=0$.

The transfer function model, which connects the observed but uncontrollable input disturbance $u_{t}$ and the output $Y_{t}$, is assumed to be

$$
y_{1 t}=\delta^{-1}(B) \omega(B) B^{b} \mu_{t}
$$

Then changes made in $X$ at times $t, t-1, t-2, \ldots$ immediately after the observations $u_{t}, u_{t-1}, u_{t-2}, \ldots$ are taken. Hence obtain a "pulsed" input, and we mark the level of $X$ in the interval $t$ to $t+1$ by $X_{t^{+}}$.

For this pulsed input, it is assumed that the transfer function model, which connects the compensating variable $X_{t}$ and the output $Y_{t}$, has the effect:

$$
y_{2 t}=L_{1}^{-1}(B) L_{2}(B) B^{f+1} X_{t+}
$$

Where $L_{1}(B)$ and $L_{2}(B)$ are polynomials in $B$.

If no control is exerted (the potential compensating variable $X_{t}$ is held fixed at $X_{t}=0$ ), the total error or deviation from target value $T=0, \varepsilon_{t}=Y_{t}-T$, the output will be:

$$
\varepsilon_{t}=\delta^{-1}(B) \omega(B) \mu_{t-b}+N_{t}
$$

The total effect of the input disturbance $(u)$ by manipulating $X_{t}$ is:

$$
\delta^{-1}(B) \omega(B) \mu_{t-b}
$$

The total effect of the input compensation (X) by manipulating $X_{t}$ is:

$$
L_{1}^{-1}(B) L_{2}(B)^{f+1} X_{t-f-1+}
$$

The control action at time $t$ should be:

$$
L_{1}^{-1}(B) L_{2}(B)^{f+1} X_{t+}=\delta^{-1}(B) \omega(B) \mu_{t-(b-f-1)}
$$

\subsection{Feedforward Control with Multiple Inputs}

The effect of several additive input disturbances $u_{1}, u_{2}, \ldots u m$ are to be compensated by changes in $X$ using feedforward control. Suppose the combined effect at the output of all the input disturbances is given by:

$$
\begin{aligned}
y_{t} & =\sum_{j=1}^{m} \delta_{j}^{-1}(B) \omega_{j}(B) B_{j}^{b} \mu_{j, t} \\
& =\sum_{j}^{m} B_{j}^{b} \mu_{j, t}^{\prime}
\end{aligned}
$$

where $\mu_{j, t}^{\prime}=(B) \omega_{j}(B) B_{j}^{b} \mu_{j, t}$,

The transfer function model for the compensating variable contributes the effect:

$$
y_{2 t}=L_{1}^{-1}(B) L_{2}(B)^{f+1} X_{t+}
$$

The required control action is to change $X$ at time $t$ by an amount:

$$
x_{t}=-L_{1}^{-1}(B) L_{1}^{-1}(B) \sum_{j=1}^{m}\left[\mu_{j, t+f+1-b_{j}}^{\prime}-\mu_{j, t+f-b_{j}}^{\prime}\right]
$$

Where

$\mu_{j, t+f+1-b_{j}}^{\prime}-\mu_{j, t+f-b_{j}}^{\prime}=\left\{\begin{array}{c}\mu_{j, t}^{\prime}\left(f+1-b_{j}\right)-\mu_{j, t-1}^{\prime}\left(+f+1-b_{j}\right) \quad f+1-b_{j}>0 \\ \mu_{j, t+f+1-b_{j}}^{\prime}-\mu_{j, t+f-b_{j}} \quad f+1-b_{j} \leq 0\end{array}\right.$

$N_{t}$ is an unmeasurable disturbance, the error or deviation from target at the output from this control action in the compensating variable $X_{l}+$ will be :

$$
\varepsilon_{t}=\sum_{j=1}^{m} e_{j, t-f-1}^{\prime}\left(f+1-b_{j}\right)+N_{t}
$$

If $f+1-b_{j} \leq 0$,

$$
e_{j, t-f-1}^{\prime}\left(f+1-b_{j}\right)=0,
$$

If $f+1-b_{j}>0$,

The forecast error corresponding to the input variable $u_{j, t}$.

Feedforward control allows us to take prompt action to cancel the effect of input disturbance variables.

To use this type of control we must be able to measure the disturbing variables and possess complete knowledge - or at least a good estimate - of the relationship between each input disturbance variable and the output.

\section{Model Building}

In fitting dynamic models, a theoretical analysis can sometimes tell us not only the appropriate form for the model, but may also provide us with good estimates of the numerical values of its parameters. These values can then be checked later by analysis of data.

The physical mechanism of a phenomenon would be theoretically to write down a mathematical expression that 
described it exactly. Thus obtain a mechanistic or theoretical model.

Use incomplete theoretical knowledge to indicate a suitable class of mathematical functions, which will then be fitted empirically; that is, the number of terms needed in the model and the numerical values of the parameters are estimated from experimental data.

The iterative approach to model building shown in Figure3.

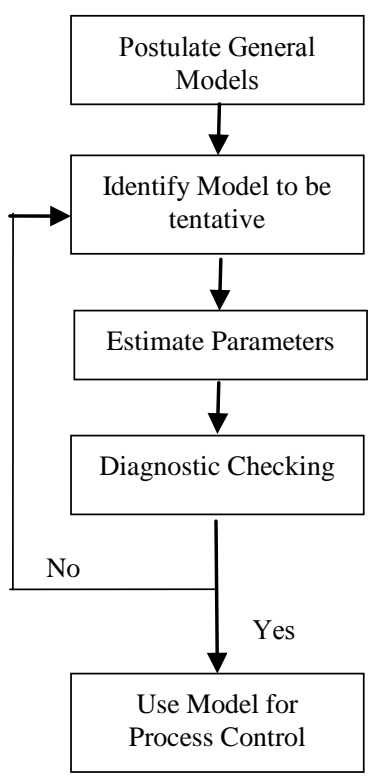

Fig. 3 Iterative approach to model building
Objective must be to obtain adequate, but parsimonious models. Control procedures could be seriously deficient if these models were either inadequate or unnecessarily prodigal in the use of parameters. Care and effort is needed in selecting the model. The process of selection is necessarily iterative; that is, it is a process of evolution, adaptation, or trial and error.

\section{References}

[1] Box G E P, Jenkins G M, Reinsel G C. Time series analysis: forecasting and control. Wiley. com, 2013.

[2] Cui You-xiang, You Jian-xin, Luo Feng Applying Fast Fourier Transform (FFT) to Statistic Quality Control, The IEEE International Conference on Industrial Engineering and Engineering Management, Macau Dec 2010: 2144-2148.

[3] Shi L. Feedforward Control and Process Improvement for Some Time Series Disturbance Models, 2013.

[4] Shi L, Kapur K C. A Synthesis of Feedback and Feedforward Control for Process Improvement Under Stationary and Nonstationary Time Series Disturbance Models. Quality and Reliability Engineering International, 2014.

[5] Coupek D, Verl A, Aichele J, et al. Proactive quality control system for defect reduction in the production of electric drives //Electric Drives Production Conference (EDPC), 2013 3rd International. IEEE, 2013: 1-6.

[6] Jiang Z Q. The Intelligent Quality Control Technology System Based on the Integration Methods of SPC and EPC. Applied Mechanics and Materials, 2013, 263: 839-842.

[7] Jelali M. Control Performance Management in Industrial Automation: Assessment, Diagnosis and Improvement of Control Loop Performance. Springer, 2013.

[8] Yang W A, Zhou W. Autoregressive coefficient-invariant control chart pattern recognition in autocorrelated manufacturing processes using neural network ensemble. Journal of Intelligent Manufacturing, 2013: 120.

[9] Little T A. Essentials in Quality Risk Management. BIOPHARM INTERNATIONAL, 2013, 26(5): 52. 